\title{
First Run II Results with the CDF Detector
}

\author{
Franco Bedeschi ${ }^{\text {a* }}$

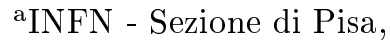 \\ via Livornese 1291 , \\ I-56010 S. Piero a Grado, Italy
}

We report the first results obtained by the CDF collaboration from the analysis of the Tevatron Run II data collected until June 2002. All components of the CDF detector are operating at or near the design specifications. Typical physics signals are observed and used both to characterize the CDF detector performance, and to make several physics measurements. In spite of the still limited accumulated luminosity some measurements are already competitive with the best currently available.

\section{Introduction}

The CDF detector has been upgraded [1] to allow operation at high luminosity and bunch spacing up to 132 nsec as planned for the Run 2 of the Tevatron. Several improvements to the Run 1 detector, which extend significantly its functionality, have also been implemented. Indeed the upgraded detector is almost all new and took many years of sustained effort to be completed.

In parallel, the Fermilab accelerator complex has also undergone a major upgrade [2]: most important a new storage ring, the Main Injector, has replaced the Main Ring as Tevatron injector and supplier of high energy protons for antiproton production. An additional new ring, the Recycler, has also been housed in the same tunnel as the Main Injector. The Recycler will allow reusing the anti-protons at the end of a store and will provide additional cooling. The goal luminosity during the first phase of Run 2 (Run 2A) is $5-8 \times 10^{31} \mathrm{~cm}^{-2} \cdot \mathrm{sec}^{-1}$ before the Recycler is fully operational and $2 \times 10^{32} \mathrm{~cm}^{-2} \cdot \mathrm{sec}^{-1}$ after full integration of the Recycler. A final integrated luminosity of $\sim 2 \mathrm{fb}^{-1}$ is planned by the end of Run $2 \mathrm{~A}$.

After a brief commissioning run in the Fall of 2000, the Run 2 officially started in March 2001. Since then much work has gone in the commissioning both of the new detectors (CDF and D0)

\footnotetext{
${ }^{*}$ On behalf of the CDF Collaboration
}

and of the accelerator complex. By February 2002 the CDF detector was in stable operation mode and had a reliable physics trigger. Data taken from that point until the start of the shutdown in early June 2002 have been used for the analyses that will be reported in this paper. This amounts to about $10-20 \mathrm{pb}^{-1}$ out of a total of $35 \mathrm{pb}^{-1}$ written to tape and $54 p b^{-1}$ delivered since the beginning of Run 2 (fig. 1. At the time of the writing of these proceedings (September 2002) the data set usable for analysis has almost tripled and a peak luminosity of $3 \times 10^{31}$ has been achieved.

\section{Upgraded CDF detector performance}

The slow components of the CDF Run 1 detector have all been upgraded to comply with the Run 2 interbunch of 132 nsec. All frontend and trigger electronics has been significantly redesigned and replaced. The Central Tracking Chamber has been replaced with a new similar chamber, the Central Outer Tracker (COT) [3], with smaller drift gaps. The Silicon Vertex Detector has been replaced with a new one, SVX2 [4], with faster readout electronics and better radiation resistance. The forward and plug gas calorimeters have been replaced by new, fast, scintillator tile based plug calorimeters.

Additional functionality has also been added to that of the Run 1 detector. The number of silicon detector layers has gone from four single 


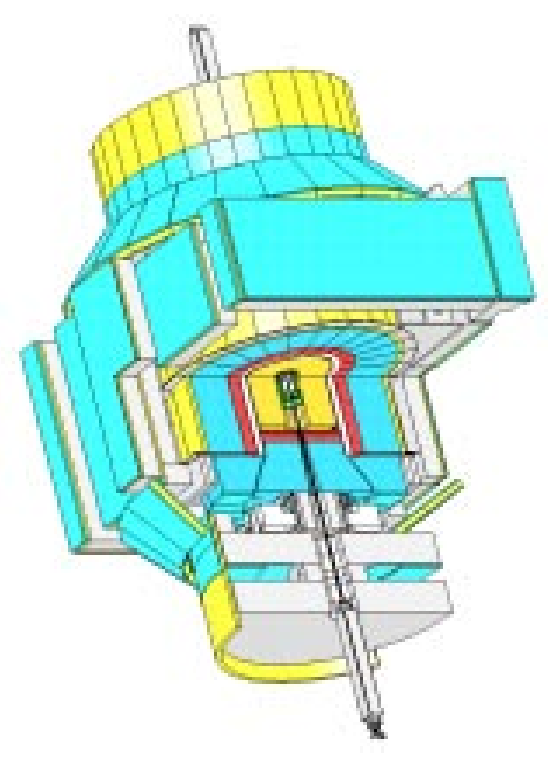

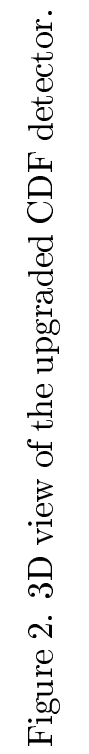

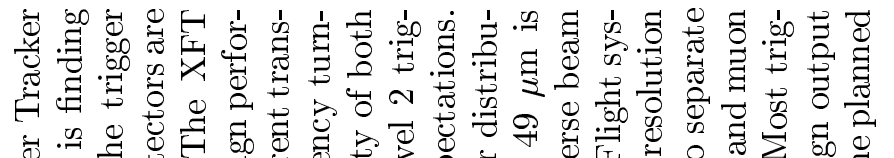

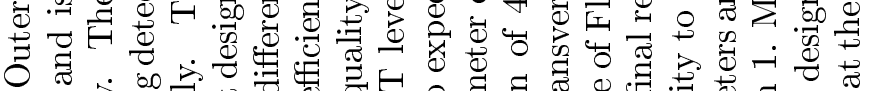
๙

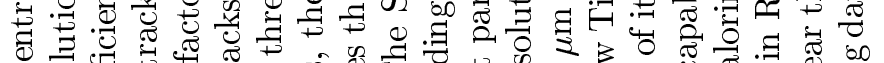

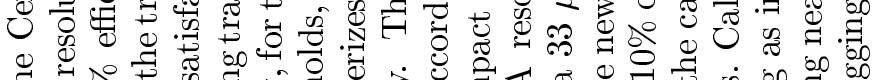
F

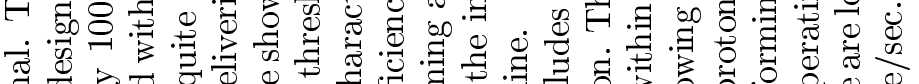

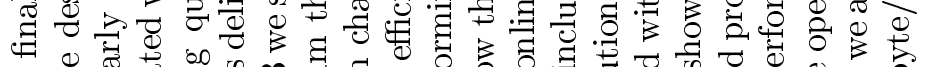

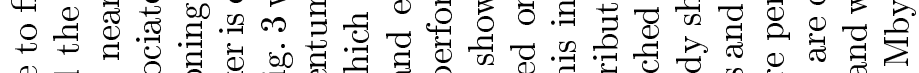

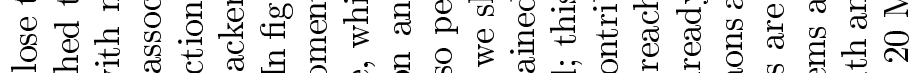

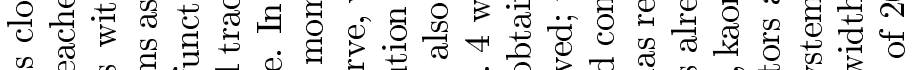
.

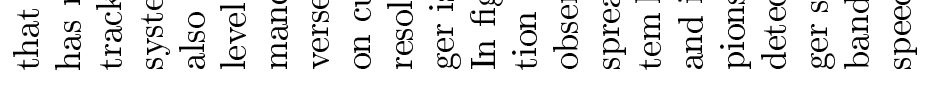
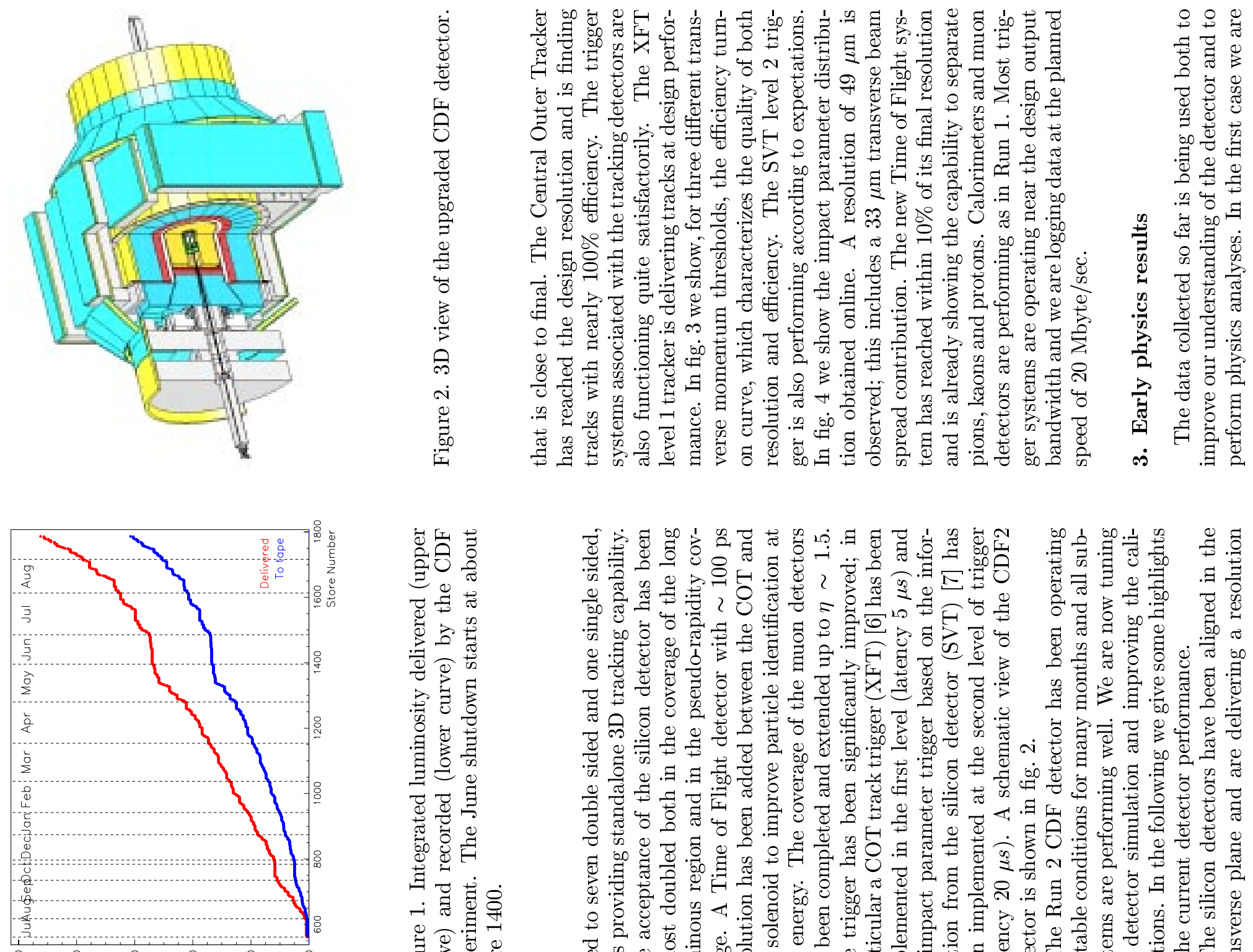

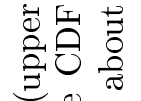

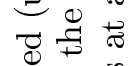

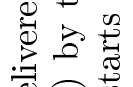

$\stackrel{\square}{\square}$

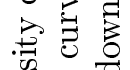

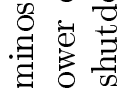

要边

च $\Xi$

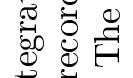

ㄷ.

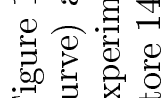

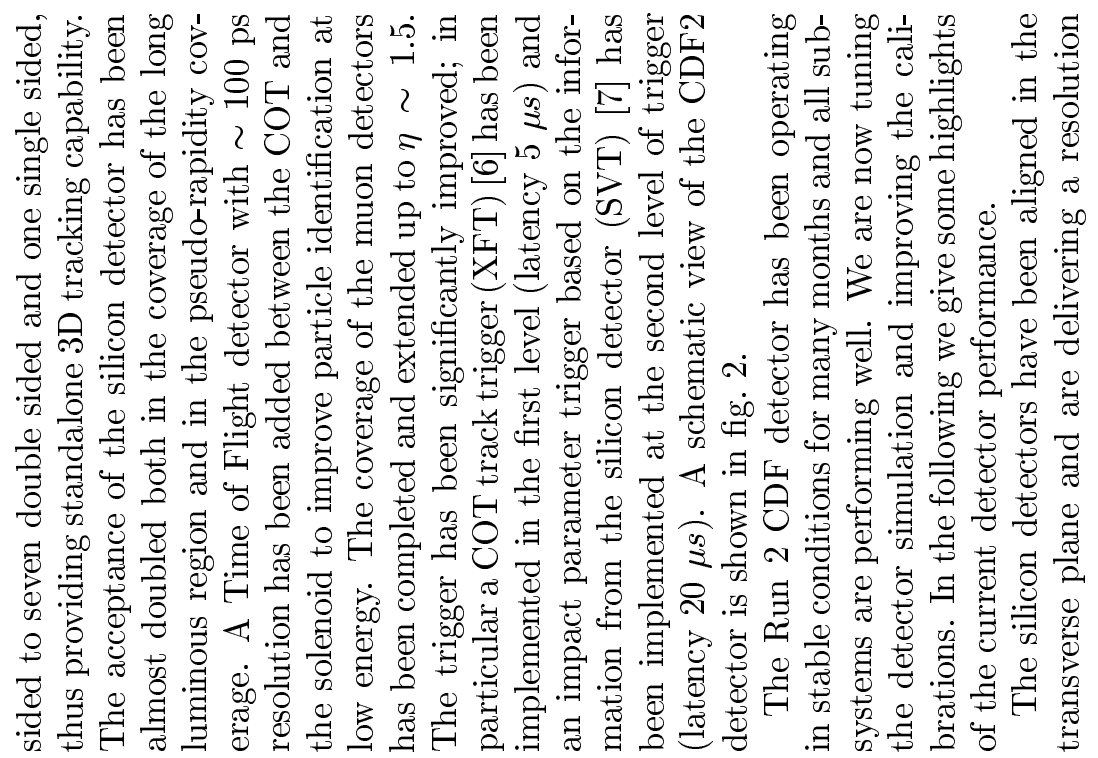



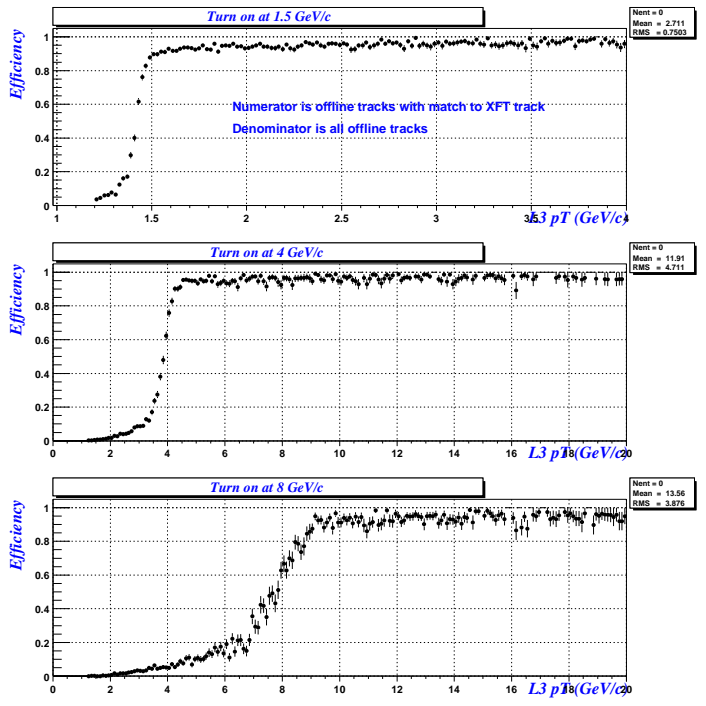

Figure 3. Efficiency turn on of the level 1 tracking trigger for some typical $p_{t}$ thresholds.

concentrating on understanding the energy scales in the calorimetry and the tracking system, on improving the calibrations, on characterizing in detail the detector performance and tuning the Monte Carlo to reproduce it. In the second case the emphasis is on doing real physics measurements with complete analyses including the full estimate of the systematic errors. This process has proved the excellent quality of our data. Even with the rather limited statistics used so far, we observe that some results are already competitive with the best measurements currently available.

In the sections that follow we discuss in more detail a selected group of early CDF results.

\subsection{Measurements with high energy elec- trons}

High energy electrons are an important signature for almost any high $E_{t}$ physics topic. We find a clear $Z \rightarrow e^{+} e^{-}$signal of 638 candidates. This sample is ideal to study the electromagnetic (EM) energy scale and the quality of our modeling of the electromagnetic calorimeters in the simulation. In fig. 5 we show the subsample of 247

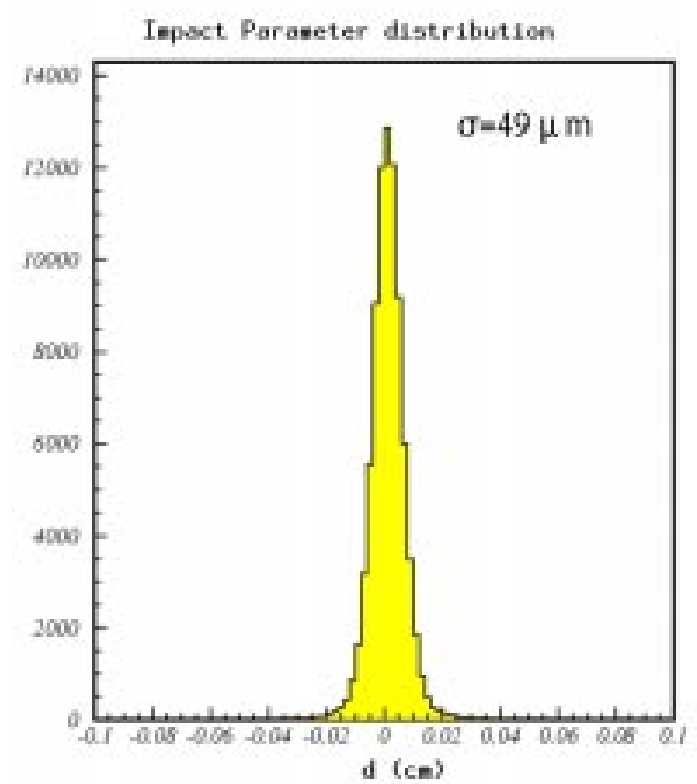

Figure 4. Online SVT trigger track impact parameter distribution.

events where both the $e^{+}$and the $e^{-}$are detected in the central calorimeter. We find that the mean value of the $Z$ mass is shifted by only $1.2 \%$ relative to the PDG value [8] using central electrons and up to $10 \%$, when also plug calorimeter electrons are included. The simulation is consistent with the PDG average and tracks the width of the $Z$ peak within $2 \%$ for central electrons and $4 \%$ for forward electrons. We are still working on improving the calibration of the new plug calorimeter. With these data we have performed a preliminary measurement of the $Z$ forward backward asymmetry as a function of the invariant mass of the $e^{+} e^{-}$pair. The result has still large errors, but it is consistent with the Standard Model expectation from $\gamma-Z$ interference.

We also look for $W$ boson decays to $e \nu$. The selection requires an isolated electron with both $E_{t}$ and missing transverse energy greater than 25 $\mathrm{GeV}$. We find 5547 candidates in $10 \mathrm{pb}^{-1}$. An accurate study of the background contributions 


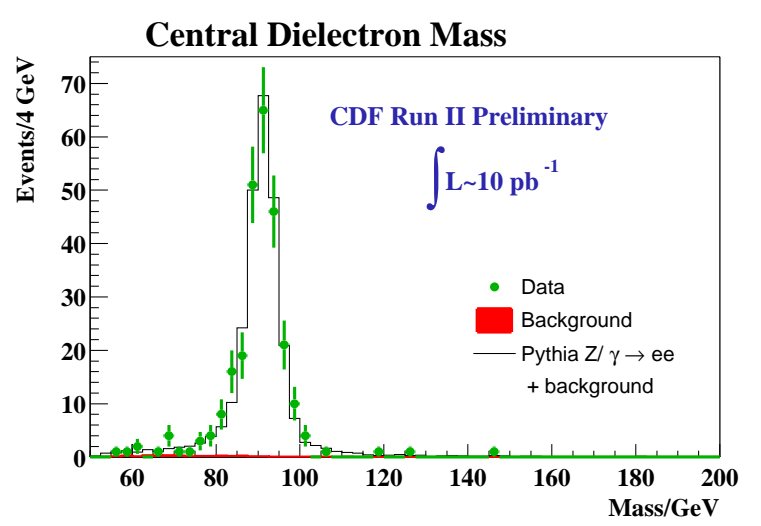

Figure 5. $Z$ peak observed in $e^{+} e^{-}$invariant mass distribution.

shows that this signal is quite clean with only about $7 \%$ of background events. We remark that the distributions of all relevant variables are modeled quite well by the sum of the W signal simulation plus background in the amounts predicted. In fig. 6 we show the transverse mass distribution as an example.

We use this sample to measure the $W$ cross section times the branching ratio to electron neutrino. The result obtained is $\sigma_{W} * B R(W \rightarrow$ $e \nu)(n b)=2.60 \pm 0.03$ (stat.) \pm 0.13 (syst.) \pm 0.26 (luminosity). This is consistent with the CDF Run 1 result [9] scaled for the ratio of the NNLO theoretical cross sections at 1800 and 1960 $\mathrm{GeV}[10]$. We note that the systematic error is already comparable with our Run 1 result. The uncertainty on the luminosity is improving fast and has already improved from $10 \%$ to $\sim 5 \%$ since the time of the ICHEP conference.

\subsection{Measurements with high energy muon samples}

Similar measurements are done with highenergy muons. In fig. 7 we show the di-muon invariant mass distribution for opposite sign central muon pairs. The selection is very stringent, as it requires the muons to be observed both in the muon chambers located just behind the cen-

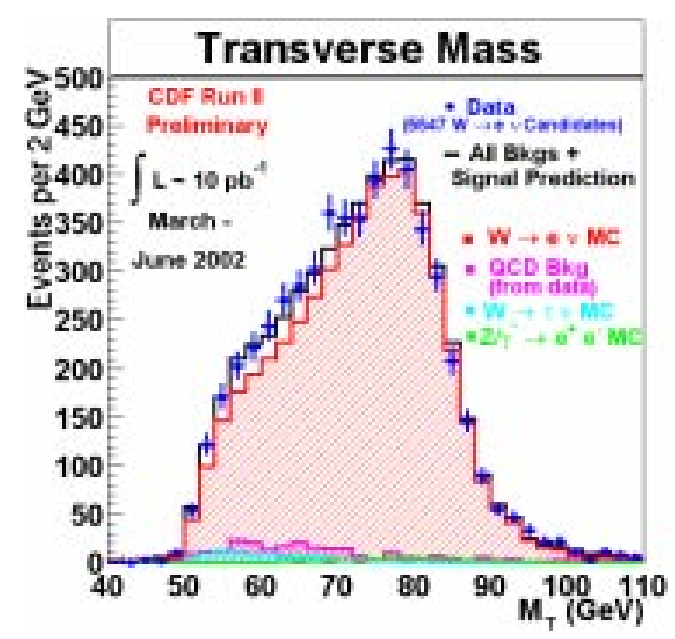

Figure 6. $W \rightarrow e \nu$ transverse mass distribution.

tral calorimeters (CMU) and, also, further out after an additional $60 \mathrm{~cm}$ of steel absorber in the CMP chambers. We observe a clean peak of 57 candidate events in $16 \mathrm{pb}^{-1}$ of data.

We also look for $W$ bosons decaying to a muon and a neutrino. We perform the selection by requiring isolated central muons with $p_{t}$ and missing transverse energy greater than $20 \mathrm{GeV}$. We find 4561 candidate events in $16 \mathrm{pb}^{-1}$ with an estimated background contamination of 569 events mostly from misidentified $Z \rightarrow \mu \mu$ and $W \rightarrow \tau \nu$. Even in this case we observe that the Monte Carlo describes quite well all relevant distributions. In fig. 8 we show the transverse mass distribution as an example.

We use this sample to measure the $W$ cross section times the branching ratio to muon neutrino. The result obtained is $\sigma_{W} * B R(W \rightarrow \mu \nu)(n b)=$ $2.70 \pm 0.04$ (stat.) \pm 0.19 (syst.) \pm 0.27 (luminosity). This is consistent with the CDF Run 1 result [11] scaled for the ratio of the NNLO theoretical cross sections at 1800 and $1960 \mathrm{GeV}$ [10].

We also measure the cross section ratio $R_{\mu}=$ $\left(\sigma_{W} * B R(W \rightarrow \mu \nu)\right) /\left(\sigma_{Z} * B R(Z \rightarrow \mu \mu)\right)$. Several systematic uncertainties are reduced in this ratio relative to the absolute cross section mea- 


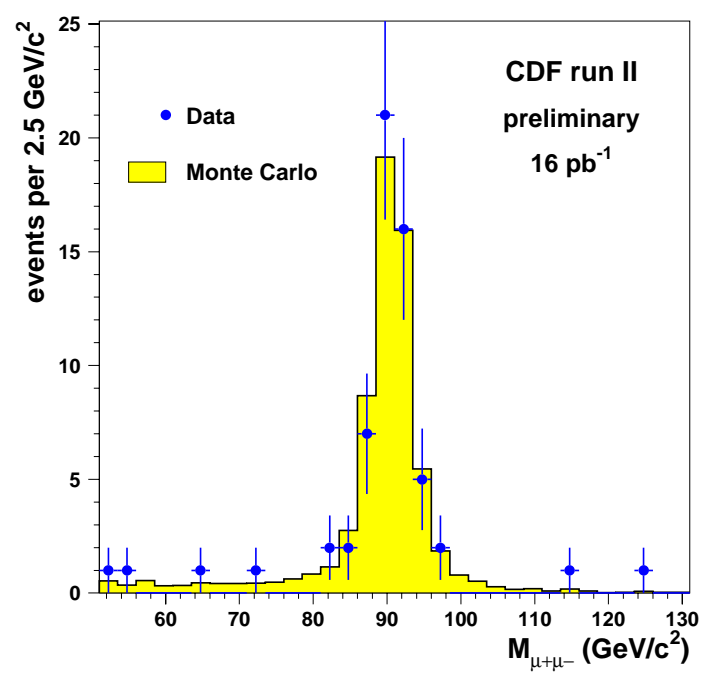

Figure 7. $Z$ peak observed in $\mu^{+} \mu^{-}$invariant mass distribution.

surements. Our result is $R_{\mu}=13.66 \pm 1.94$ (stat.) \pm 1.16 (syst.). This is consistent, within the large error, with our Run 1 result [11] after scaling for the difference in beam energy.

\subsection{Measurements with low energy muons}

The CDF samples of low energy muons have played an important role for $\mathrm{B}$ and onia physics during Run 1. The trigger improvements installed for Run 2, in particular the Level 1 tracking trigger, has improved the muon quality therefore allowing a reduction in the muon candidate $p_{t}$ threshold from 2.0 to $1.5 \mathrm{GeV} / \mathrm{c}$ for di-muon triggers. In the case of the $J / \psi$ trigger the minimum track separation has also been reduced from 5 to 2.5 degrees.

In fig. 9 we show the currently available signal of 107 thousand $J / \psi$ in $13 p b^{-1}$. This sample is reduced by about a factor two when both muons are required to be reconstructed also in the vertex detector. This observed rate of $J / \psi$ 's and the mass resolution $\left(21 \mathrm{MeV} / \mathrm{c}^{2}\right.$ with only COT information and $15 \mathrm{MeV} / \mathrm{c}^{2}$ when the silicon detec-

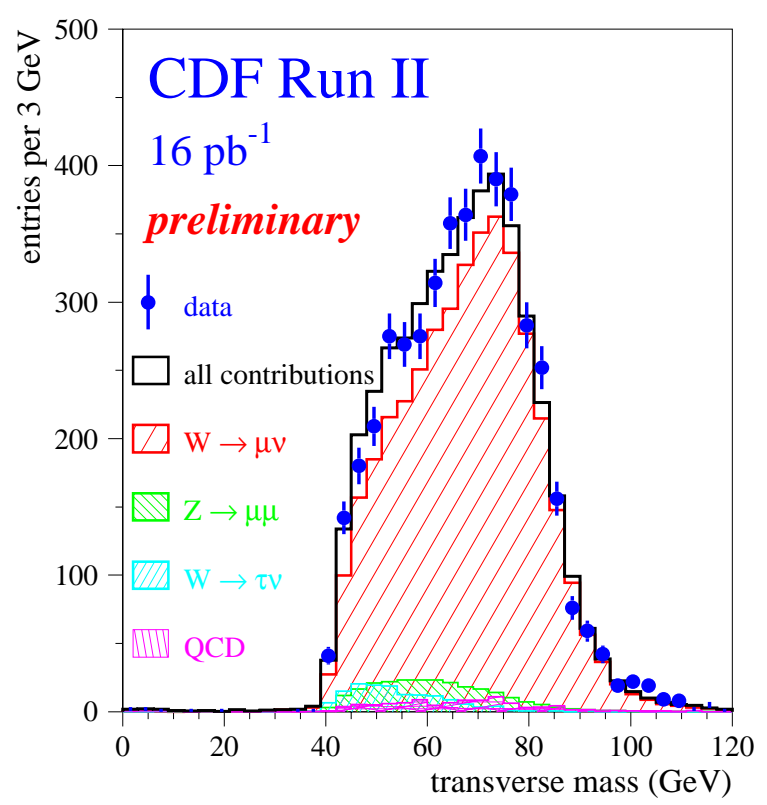

Figure 8. $W \rightarrow \mu \nu$ transverse mass distribution.

tor information is included) are consistent with expectations.

While most $J / \psi$ 's produced at the Tevatron are prompt, a fair fraction originates from decays of B mesons. Our sample is large enough to allow us to statistically separate the two components and measure both the inclusive b-hadron lifetime and the fraction of prompt $J / \psi$ 's. We obtain $c \tau=$ $458 \pm 10$ (stat.) \pm 11 (syst.) $\mu \mathrm{m}$. This result is consistent both with the PDG value [8] and with previous CDF measurements [12]. This is a good indication that we have most tracking systematics well under control. While the statistical uncertainty is still high relative to our Run 1 measurement [12], the systematic error is already better. The measured prompt fraction returned by the lifetime fit is $83 \%$; this is larger than the Run 1 value as expected from the lowering of the trigger $p_{t}$ threshold. This large prompt component is useful to test the quality of our understanding of the tracking errors. We find that the standard deviation of the observed transverse 


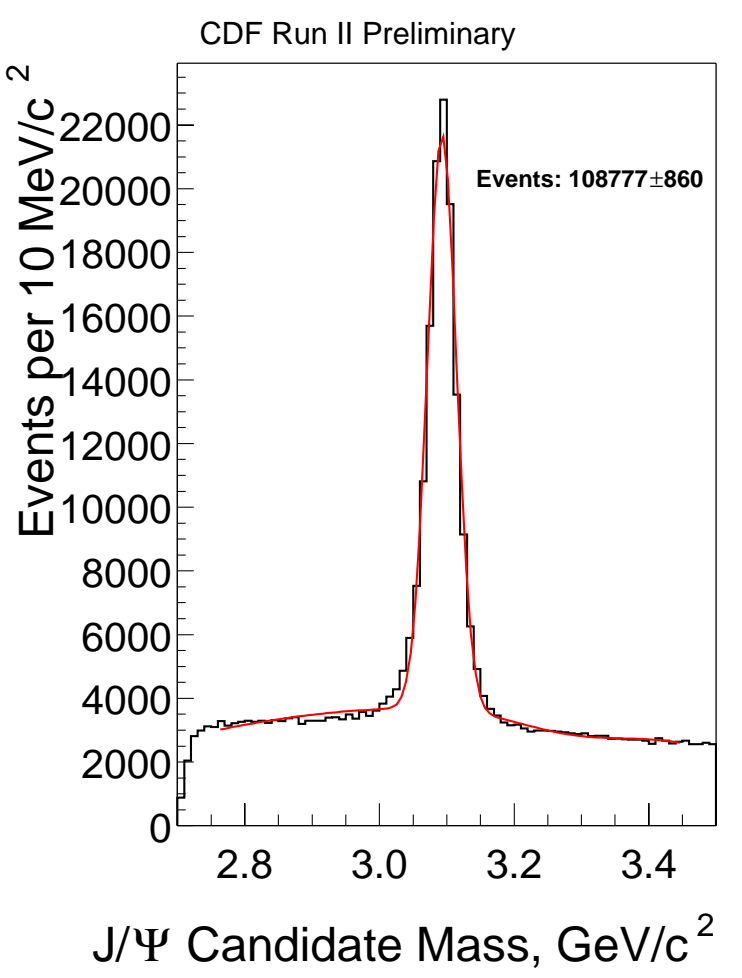

Figure 9. $J / \psi$ peak observed in $\mu^{+} \mu^{-}$invariant mass distribution.

life-path distribution is just $7 \%$ larger than the calculated errors.

The $J / \psi$ sample is also used to calibrate the corrections to the tracking momentum scale for the $\mathrm{dE} / \mathrm{dx}$ loss in the material transversed and for the value of the magnetic field. In fig. 10 we show the dependence of the average reconstructed $J / \psi$ mass as a function of the $J / \psi p_{t}$. The lowest set of points shows what we observe before any correction is applied: there is a clear slope and an offset relative to the PDG value of the $J / \psi$ mass [8]. Taking into account $\mathrm{dE} / \mathrm{dx}$ losses as described in our GEANT simulation reduces the slope and shifts the line upward, but we need to assume an additional $20 \%$ more material to eliminate the slope completely. This is

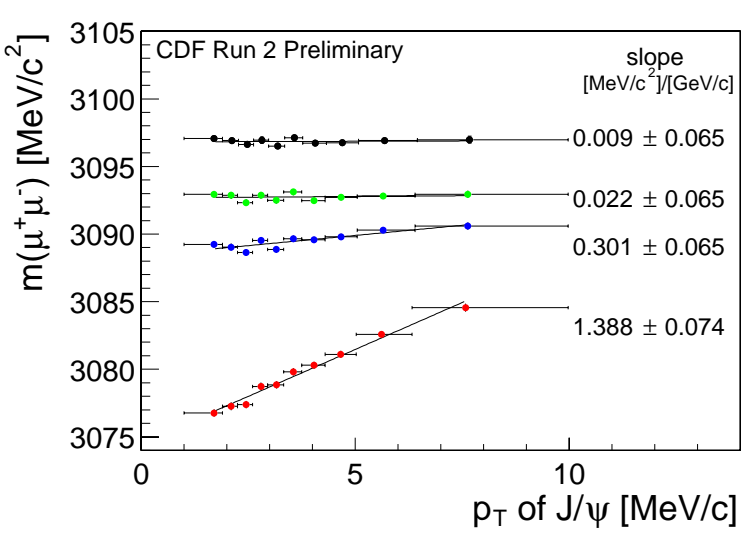

Figure 10. Material and B field calibration using $J / \psi$ 's.

consistent with an independent analysis of the frequency and distribution of photon conversions in the CDF detector. Finally a magnetic field correction is applied to shift the data to the upper band in fig. 10. We have applied these corrections to independent high statistics samples like $D^{0} \rightarrow K \pi$ and $\Upsilon \rightarrow \mu \mu$ and find that they yield an excellent consistency with the expected mass values. After this calibration the quality of our momentum scale is at the level of $0.02 \%$.

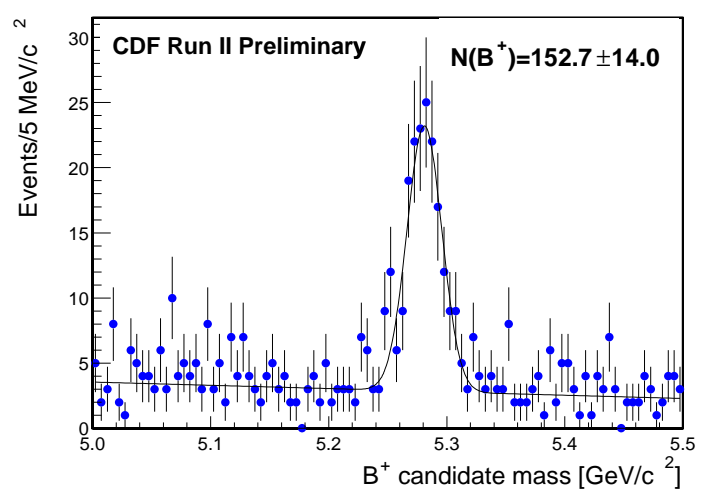

Figure 11. Observed $B^{+} \rightarrow J / \psi K^{+}$peak. 
Table 1

Measured $B$ meson masses compared to current PDG [8] values.

\begin{tabular}{lllcc}
\hline B meson type & Decay mode & Mass $\left(\mathrm{MeV} / \mathrm{c}^{2}\right)$ & $\Delta P D G / \sigma(C D F)$ & $\sigma(C D F) / \sigma(P D G)$ \\
\hline$B_{u}^{+}$ & $J / \psi K^{+}$ & $5280.60 \pm 1.70 \pm 1.1$ & 0.77 & 4.05 \\
$B_{d}^{0}$ & $J / \psi K^{0 *}$ & $5279.80 \pm 1.90 \pm 1.4$ & 0.17 & 4.72 \\
$B_{s}^{0}$ & $J / \psi \varphi$ & $5360.30 \pm 3.80 \pm 2.10$ & -1.81 & 1.90 \\
\hline
\end{tabular}

Relying on this momentum calibration we measure the masses of the B mesons using some of their exclusive decays modes including $J / \psi$ 's. In fig. 11 we show the invariant mass distribution of the $J / \psi K^{+}$candidates as an example. A clear $B^{+}$signal of 153 events is observed in $18.3 \mathrm{pb}^{-1}$ of data. Table 1 summarizes all of our recent results. In the fourth column we show the difference with the current world average divided by the our combined statistical and systematic error. We observe a good consistency. In the fifth column we compare our combined error with the current PDG uncertainty and find that that, in spite of the very limited statistics, we are typically just a factor 4-5 worse than the current world average.

\subsection{Hadronic jets}

Hadronic jets are one of the key signatures for Hadron Collider physics. Since the start of Run $2 \mathrm{CDF}$ has already collected millions of jet events up to uncorrected energies of about $400 \mathrm{GeV}$. Before they can be used effectively in many analysis, however, we need to establish a good understanding of the hadronic energy scale as well as several corrections to the raw jet energy, some of which depend on having a reliable simulation of the jet shapes.

We use muons from $J / \psi$ decays to probe the response of the hadronic calorimeters to minimum ionizing particles. We find that the peak of the ionization peak is $4 \%$ lower than during the previous run in the central calorimeter, which is well covered by the $J / \psi$ trigger. This deviation is supported by an independent analysis using events with one photon and one jet only and checking the balance of the transverse energy.

Jet shapes are studied by comparing energy flows in cones of various sizes to a simulation based on Herwig followed by full detector sim-

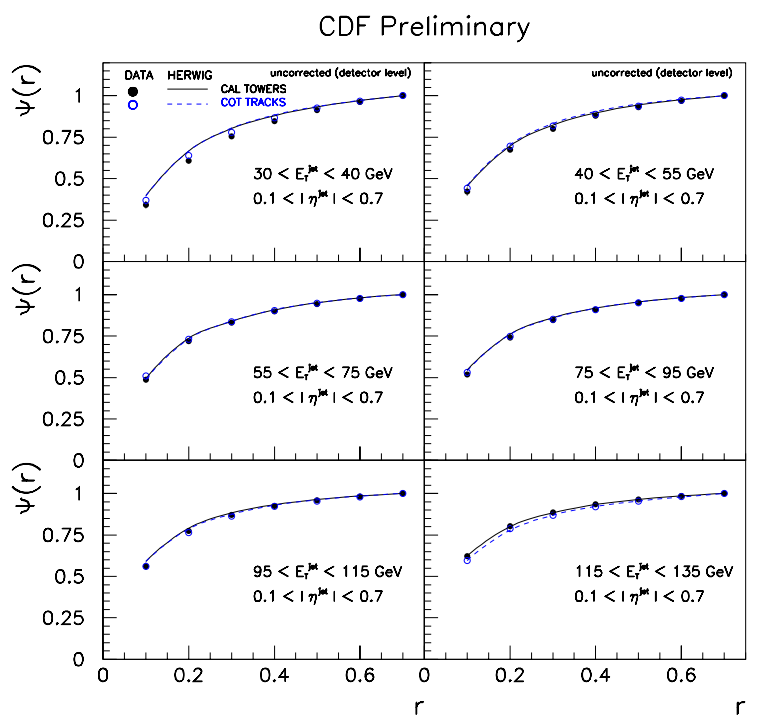

Figure 12. Fraction of the jet energy contained in an $\eta-\varphi$ cone as a function of its radius, $\mathrm{r}$. Results are shown for several jet energy bins.

ulation. We find jets using standard cone clustering algorithms applied both to calorimeter towers and, alternatively, to tracks. We observe a remarkable agreement between data and simulation in both cases as shown in fig. 12 .

\subsection{Secondary vertex triggers}

The trigger on secondary vertices has opened a whole new way of studying beauty and charm quark physics at a Hadron Collider. Our trigger currently selects two tracks with at least $2 \mathrm{GeV}$ at Level 1 and then requires an impact parameter greater than $100 \mu \mathrm{m}$ at Level 2. For the twobody decay trigger path additional pointing and kinematical constraints are required, while for the 
multi-body decay path the impact parameter cut is raised to $120 \mu \mathrm{m}$.

We observe that this trigger is very efficient to select events with charmed mesons. Indeed the yield of $D^{0} \rightarrow K^{-} \pi^{+}$is comparable to that of $J / \psi$ 's. In fig. 13 we show the $D^{0}$ signal in 10 $p b^{-1}$ containing over 50 thousand events. We use this signal to monitor the stability of the trigger online during data taking.

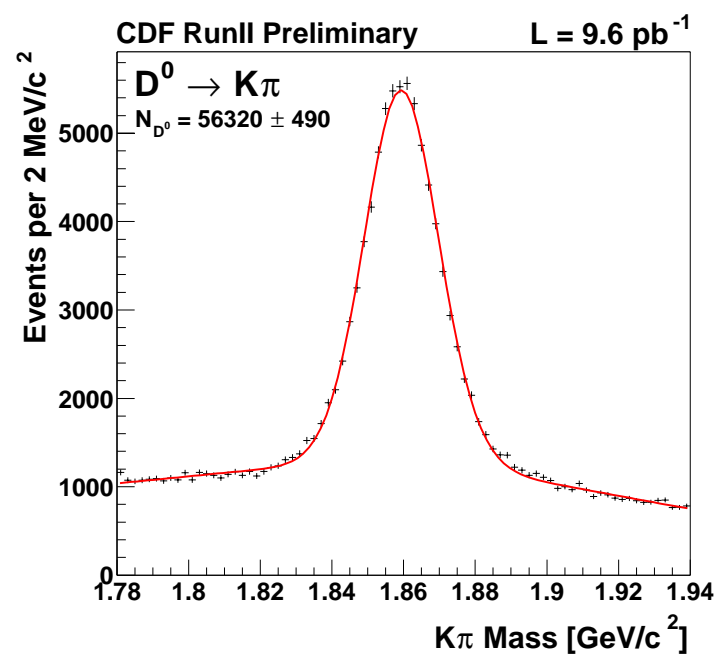

Figure 13. $D^{0} \rightarrow K^{-} \pi^{+}$candidate invariant mass distribution.

Large amounts of Cabibbo suppressed $D^{0}$ decays into $K^{+} K^{-}(5670$ events $)$ and $\pi^{+} \pi^{-}$ (2020 events) are also observed. An example is shown in fig. 14. We use these signals to measure the branching ratios of these decay modes relative to the $D^{0} \rightarrow K \pi$ mode. We find $\operatorname{BR}\left(D^{0} \rightarrow K^{+} K^{-}\right) / \mathrm{BR}\left(D^{0} \rightarrow\right.$ $\left.K^{-} \pi^{+}\right)=(11.17 \pm 0.48 \pm 0.98) \%$ and $\operatorname{BR}\left(D^{0} \rightarrow\right.$ $\left.\pi^{+} \pi^{-}\right) / \mathrm{BR}\left(D^{0} \rightarrow K^{-} \pi^{+}\right)=(3.37 \pm 0.20 \pm 0.16) \%$ in good agreement with the world average [8] and with comparable resolution.

Other $D$ species are also observed in these displaced vertex trigger samples as shown in fig. 15 . For instance the $D^{+}(1350$ events $)$ and $D_{s}^{+}(2360$

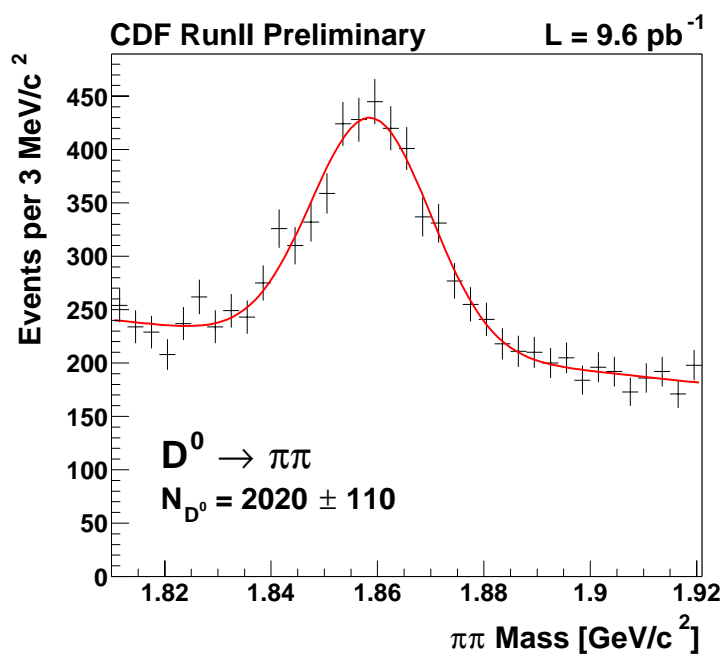

Figure 14. $D^{0} \rightarrow \pi^{-} \pi^{+}$candidate invariant mass distribution.

events), which we identify using their common decay into $\varphi \pi$. The common selection and decay kinematics make this sample ideal to measure their mass difference. We find $m\left(D_{s}^{+}\right)-m\left(D^{+}\right)$ $=99.28 \pm 0.43 \pm 0.27 \mathrm{MeV} / \mathrm{c}^{2}$. This result is consistent with the world average [8] and has approximately the same resolution in spite of being still statistically limited.

It is interesting to understand if the main source of all this charm is direct $c \bar{c}$ production or else it originates mostly from the decay of bhadrons. We have started to understand this by studying the impact parameter of the $D$ meson relative to the primary interaction vertex. We expect that $D$ mesons from $B$ decays would show large non-gaussian tails in the $D$ impact parameter distribution. We find that $\sim 85 \%$ of nonstrange $D$ 's and $\sim 65 \%$ of $D_{s}$ come from direct production.

We also look for fully hadronic B meson decays. This is harder because the two body decays have very small branching fractions and the multibody decays have a larger combinatoric background. We observe a clear $B^{+} \rightarrow D^{0} \pi^{+}$signal of 56 events in $10 p b^{-1}$ as shown in fig. 16. We also 


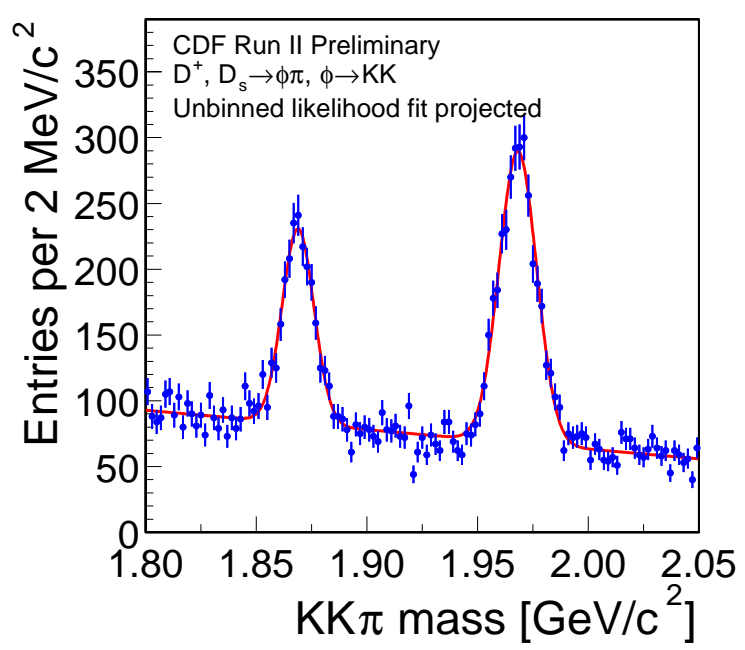

Figure 15. $D_{s}^{+} / D^{+} \rightarrow \varphi \pi^{+}$candidate invariant mass distribution.

see for the first time at a Hadron Collider a signal of $B$ decays to two hadrons. In fig. 16 we show the observed signal peak consisting of 33 events. The peak is wider than our mass resolution as it is the sum of several contributions: $B_{d}^{0} \rightarrow \pi^{+} \pi^{-}$, $B_{d}^{0} \rightarrow K^{+} \pi^{-}, B_{s}^{0} \rightarrow K^{-} \pi^{+}, B_{s}^{0} \rightarrow K^{+} K^{-}$. The plotted invariant mass has been calculated assuming always the pion mass. We note that, while these first observations of hadronic $B$ meson decays are exciting, the number of events observed is smaller than expected. We understand this discrepancy well with a simulation which accounts for a detailed description of the SVT trigger and the actual silicon detector coverage. This coverage has been steadily improving and after the shutdown of June 2002 has been increased to more than $90 \%$. The SVT firmware optimization has also progressed bringing the track finding efficiency from 80 to $90 \%$. All in all we expect a factor 3 improvement in hadronic B trigger rates. Additional improvements are expected from the track reconstruction software. We also notice that the signal to noise ratio obtained for the $B \rightarrow h^{+} h^{-}$signal is much better than our most optimistic expectations. This partially compensates for the reduced statistics in any asymmetry measurement based on these data.

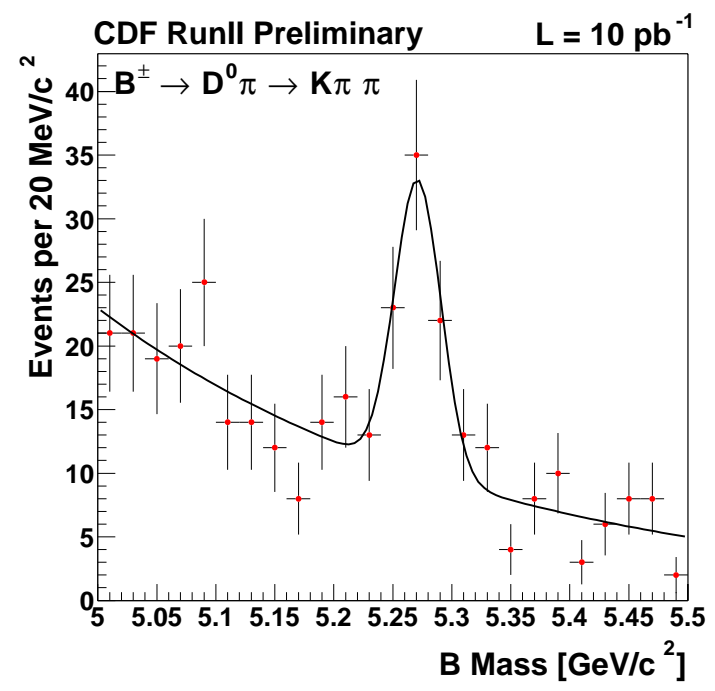

Figure 16. $B^{+} \rightarrow D^{0} \pi^{+}$candidate invariant mass distribution.

\section{Conclusions}

The CDF detector is clearly back into operation and taking good quality data with all of its sub-systems fully functional. The understanding of the detector is very advanced and is progressing much faster than planned. This leads to many physics analysis, which the CDF collaboration has often carried all the way to the final result. Surprisingly enough some of these results have proved to be already competitive in spite of the rather limited integrated luminosity so far analyzed. In any case these analyses have made us ready to exploit more statistics when it will become available. Furthermore they show that the upgraded CDF detector is more powerful than the previous one and has an excellent potentiality for high quality physics as soon as the integrated luminosity will grow to the expected levels. 


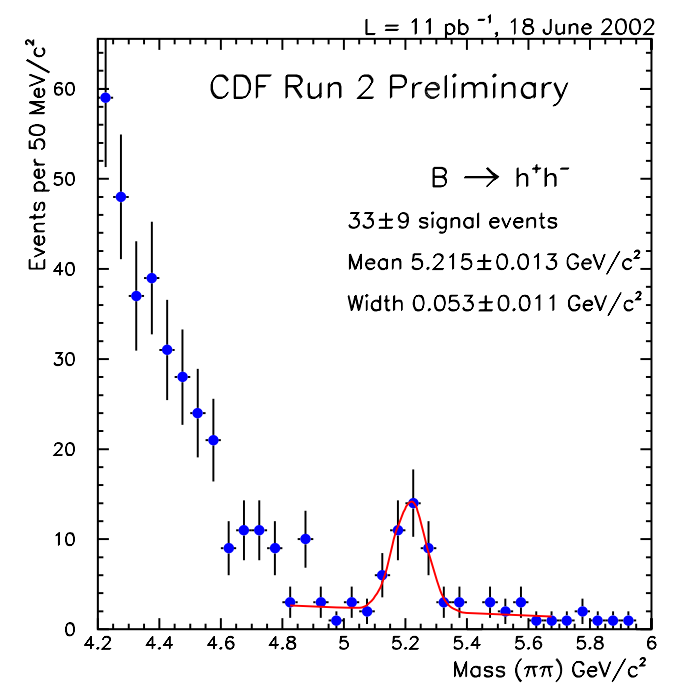

F. Abe et al., Phys. Rev. Lett. 71 (1993) 3421.

Figure 17. $B^{0} \rightarrow h^{-} h^{+}$candidate invariant mass distribution.

\section{REFERENCES}

1. CDF Collaboration, "The CDF II Detector Technical Design Report", FERMILAB-Pub96/390-E.

2. S. D. Holmes, Proceedings of the XV International Conference on High Energy Accelerators, World Scientific (1992) 34.

3. Nucl. Instrum. and Meth. A461 (2001)62.

4. Nucl. Instrum. and Meth. A447 (2000) 1.

5. Nucl. Instrum.Meth. A480 (2002) 524 .

6. S. Holm et al., IEEE Trans. Nucl. Sci. 47 (2000) 895.

7. A. Bardi et al., Nucl. Instrum. Meth. A409 (1998) 658.

8. K. Hagiwara et al., Phys. Rev. D66 (2002) 010001.

9. F. Abe et al., Phys. Rev. Lett. 76 (1996) 3070.

10. A. D. Martin et al., Phys.Lett. B531 (2002) 216 ;

W. J. Stirling: Private communication.

11. F. Abe et al., Phys. Rev. Lett. 69 (1992) 28.

12. F. Abe et al., Phys. Rev. D57 (1998) 5382; 